\title{
Context-Sensitive Lexicon Features for Neural Sentiment Analysis
}

\author{
Zhiyang Teng, Duy-Tin Vo and Yue Zhang \\ Singapore University of Technology and Design \\ \{zhiyang_teng, duytin_vo\}@mymail.sutd.edu.sg \\ yue_zhangesutd.edu.sg
}

\begin{abstract}
Sentiment lexicons have been leveraged as a useful source of features for sentiment analysis models, leading to the state-of-the-art accuracies. On the other hand, most existing methods use sentiment lexicons without considering context, typically taking the count, sum of strength, or maximum sentiment scores over the whole input. We propose a context-sensitive lexicon-based method based on a simple weighted-sum model, using a recurrent neural network to learn the sentiments strength, intensification and negation of lexicon sentiments in composing the sentiment value of sentences. Results show that our model can not only learn such operation details, but also give significant improvements over state-of-the-art recurrent neural network baselines without lexical features, achieving the best results on a Twitter benchmark.
\end{abstract}

\section{Introduction}

Sentiment lexicons (Hu and Liu, 2004; Wilson et al., 2005; Esuli and Sebastiani, 2006) have been a useful resource for opinion mining (Kim and Hovy, 2004; Agarwal et al., 2011; Moilanen and Pulman, 2007; Choi and Cardie, 2008; Mohammad et al., 2013; Guerini et al., 2013; Vo and Zhang, 2015). Containing sentiment attributes of words such as polarities and strengths, they can serve to provide a word-level foundation for analyzing the sentiment of sentences and documents. We investigate an effective way to use sentiment lexicon features.

A traditional way of deciding the sentiment of a document is to use the sum of sentiment values of

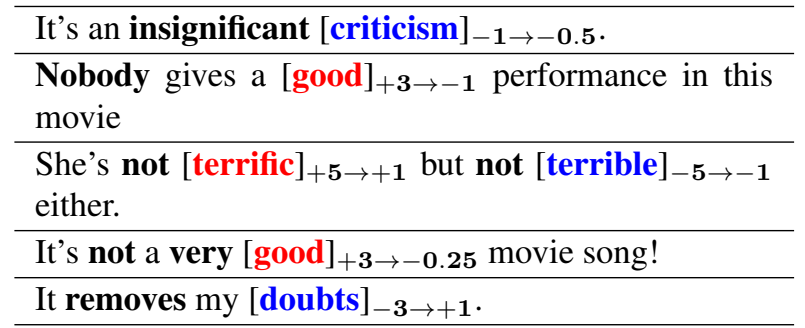

Figure 1: Example sentiment compositions.

all words in the document that exist in a sentiment lexicon (Turney, 2002; Hu and Liu, 2004). This simple method has been shown to give surprisingly competitive accuracies in several sentiment analysis benchmarks (Kiritchenko et al., 2014), and is still the standard practice for specific research communities with mature domain-specific lexicons, such as finance (Kearney and Liu, 2014) and product reviews (Ding et al., 2008).

More sophisticated sentence-level features such as the counts of positive and negative words, their total strength, and the maximum strength, etc, have also been exploited (Kim and Hovy, 2004; Wilson et al., 2005; Agarwal et al., 2011). Such lexicon features have been shown highly effective, leading to the best accuracies in the SemEval shared task (Mohammad et al., 2013). On the other hand, they are typically based on bag-of-word models, hence suffering two limitations. First, they do not explicitly handle semantic compositionality (Polanyi and Zaenen, 2006; Moilanen and Pulman, 2007; Taboada et al., 2011), some examples of which are shown in Figure 1. The composition effects can exhibit intricacies such as negation over intensification (e.g. not very good), shifting (e.g. not terrific) vs flip- 
ping negation (e.g. not acceptable), content word negation (e.g. removes my doubts) and unbounded dependencies (e.g. No body gives a good performance).

Second, they cannot effectively deal with word sense variations (Devitt and Ahmad, 2007; Denecke, 2009). Guerini et al. (2013) show challenges in modeling the correlation between contextdependent posterior word sentiments and their context independent priors. For example, the sentiment value of "cold" varies between "cold beer", "cold pizza" and "cold person" due to sense and context differences. Such variations raise difficulties for a sentiment classifier with bag-of-word nature, since they can depend on semantic information over long phrases or the full sentence.

We investigate a method that can potentially address the above issues, by using a recurrent neural network to capture context-dependent semantic composition effects over sentences. Shown in Figure 2, the model is conceptually simple, using a weighted sum of lexicon sentiments and a sentence-level bias to estimate the sentiment value of a sentence. The key idea is to use a bi-directional long-short-term-memory (LSTM) (Hochreiter and Schmidhuber, 1997; Graves et al., 2013) model to capture global syntactic dependencies and semantic information, based on which the weight of each sentiment word together with a sentence-level sentiment bias score are predicted. Such weights are context-sensitive, and can express flipping negation by having negative values.

The advantages of the recurrent network model over existing semantic-composition-aware discrete models such as (Choi and Cardie, 2008) include its capability of representing non-local and subtle semantic features without suffering from the challenge of designing sparse manual features. On the other hand, compared with neural network models, which recently give the state-of-the-art accuracies ( $\mathrm{Li}$ et al., 2015; Tai et al., 2015), our model has the advantage of leveraging sentiment lexicons as a useful resource. To our knowledge, we are the first to integrate the operation into sentiment lexicons and a deep neural model for sentiment analysis.

The conceptually simple model gives strong empirical performances. Results on standard sentiment benchmarks show that our method gives competitive

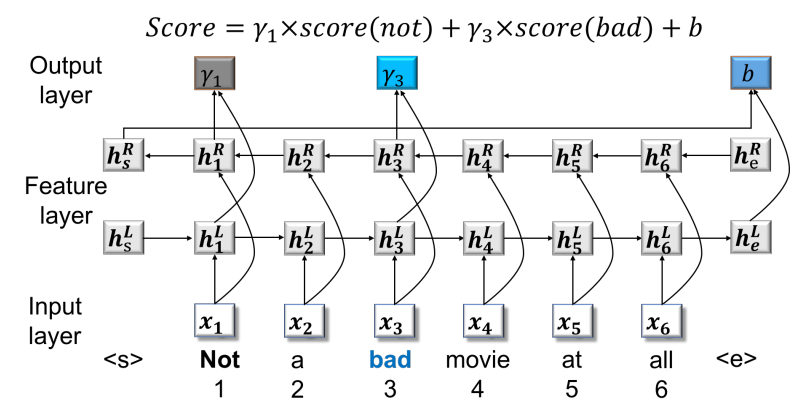

Figure 2: Overall model structure. The sentiment score of the sentence "not a bad movie at all" is a weighted sum of the scores of sentiment words "not", "bad" and a sentence-level bias score b. score (not) and score(bad) are prior scores obtained from sentiment lexicons. $\gamma_{1}$ and $\gamma_{3}$ are context-sensitive weights for sentiment words "not" and "bad", respectively.

accuracies to the state-of-the-art models in the literature. As a by-product, the model can also correctly identify the compositional changes on the sentiment values of each word given a sentential context.

Our code is released at https://github.com/zeeeyang/lexicon_rnn.

\section{Related Work}

There exist many statistical methods that exploit sentiment lexicons (Kim and Hovy, 2004; Agarwal et al., 2011; Mohammad et al., 2013; Guerini et al., 2013; Tang et al., 2014b; Vo and Zhang, 2015; Cambria, 2016). Mohammad et al. (2013) leverage a large sentiment lexicon in a SVM model, achieving the best results in the SemEval 2013 benchmark on sentence-level sentiment analysis (Nakov et al., 2013). Compared to these methods, our model has two main advantages. First, we use a recurrent neural network to model context, thereby exploiting non-local semantic information. Second, our model offers context-sensitive operational details on each word.

Several previous methods move beyond bag-ofword models in leveraging lexicons. Most notably, Moilanen and Pulman (2007) introduce the ideas from compositional semantics (Montague, 1974) into sentiment operations, developing a set of composition rules for handling negations. Along the line, Taboada et al. (2011) developed a lexicon and a collection of sophisticated rules for addressing intensification, negation and other phenomena. Differ- 
ent from these rule-based methods, Choi and Cardie (2008) use a structured linear model to learn semantic compositionality relying on a set of manual features. In contrast, we leverage a recurrent neural model for inducing semantic composition features automatically. Our weighted-sum representation of semantic compositionality is formally simpler compared with fine-grained rules such as (Taboada et al., 2011). However, it is sufficient for describing the resulting effect of complex and context-dependent operations, with the semantic composition process being modeled by LSTM. Our sentiment analyzer also enjoys a more competitive LSTM baseline compared to a traditional discrete models.

Our work is also related to recent work on using deep neural networks for sentence-level sentiment analysis, which exploits convolutional (Kalchbrenner et al., 2014; Kim, 2014; Ren et al., 2016), recursive (Socher et al., 2013; Dong et al., 2014; Nguyen and Shirai, 2015) and recurrent neural networks (Liu et al., 2015; Wang et al., 2015; Zhang et al., 2016), giving highly competitive accuracies. As our baseline, LSTM (Tai et al., 2015; Li et al., 2015) stands among the best neural methods. Our model is different from these prior methods in mainly two aspects. First, we introduce sentiment lexicon features, which effectively improve classification accuracies. Second, we learn extra operation details, namely the weights on each word, automatically as hidden variables. While the baseline uses LSTM features to perform end-to-end mapping between sentences and sentiments, our model uses them to induce the lexicon weights, via which word level sentiment are composed to derive sentence level sentiment.

\section{Model}

Formally, given a sentence $s=w_{1} w_{2} \ldots w_{n}$ and a sentiment lexicon $D$, denote the subjective words in $s$ as $w_{j_{1}}^{D} w_{j_{2}}^{D} \ldots w_{j_{m}}^{D}$. Our model calculates the sentiment score of $s$ according to $D$ in the form of

$$
\operatorname{Score}(s)=\sum_{t=1}^{m} \gamma_{j_{t}} \operatorname{score}\left(w_{j_{t}}^{D}\right)+b,
$$

where $\operatorname{Score}\left(w_{j_{t}}^{D}\right)$ is the sentiment value of $w_{j_{t}}, \gamma_{j_{t}}$ are sentiment weights and $b$ is a sentence-level bias. The sentiment values of words and sentences are real numbers, with the sign indicating the polarity and the absolute value indicating the strength.

As shown in Figure 2, our neural model consists of three main layers, namely the input layer, the feature layer and the output layer. The input layer maps each word in the input sentence into a dense real-value vector. The feature layer exploits a bidirectional LSTM (Graves and Schmidhuber, 2005; Graves et al., 2013) to extract non-local semantic information over the sequence. The output layer calculates a weight score for each sentiment word, as well as an overall sentiment bias of the sentence.

In this figure, the score of the sentence "not a bad movie at all" is decided by a weighted sum of the sentiments of "bad" and "not"1, and a sentiment shift bias based on the sentence structure. Ideally, the weight on "not" should be a small negative value, which results in a slightly positive sentiment shift. The weight on "bad" should be negative, which represents a flip in the polarity. These weights jointly model a negation effect that involves both shifting and flipping.

\subsection{Bidirectional LSTM}

We use LSTM (Hochreiter and Schmidhuber, 1997) for feature extraction, which recurrently processes sentence $s$ token by token. For each word $w_{t}$, the model calculate a hidden state vector $\mathbf{h}_{\mathbf{t}}$. A LSTM cell block makes use of an input gate $\mathbf{i}_{t}$, a memory cell $\mathbf{c}_{t}$, a forget gate $f_{t}$ and an output gate $\mathbf{o}_{t}$ to control information flow from the history $\mathrm{x}_{1} \ldots \mathrm{x}_{\mathrm{t}}$ and $\mathbf{h}_{\mathbf{1}} \ldots \mathbf{h}_{\mathbf{t}-\mathbf{1}}$ to the current state $\mathbf{h}_{\mathbf{t}}$. Formally, $\mathbf{h}_{\mathbf{t}}$ is computed as follows:

$$
\begin{aligned}
& \mathbf{i}_{\mathbf{t}}=\sigma\left(\mathbf{W}_{\mathbf{i}} \mathbf{x}_{\mathbf{t}}+\mathbf{U}_{\mathbf{i}} \mathbf{h}_{\mathbf{t}-\mathbf{1}}+\mathbf{V}_{\mathbf{i}} \mathbf{c}_{\mathbf{t}-\mathbf{1}}+\mathbf{b}_{\mathbf{i}}\right) \\
& \mathbf{f}_{\mathbf{t}}=\mathbf{1 . 0}-\mathbf{i}_{\mathbf{t}} \\
& \mathbf{g}_{\mathbf{t}}=\tanh \left(\mathbf{W}_{\mathbf{g}} \mathbf{x}_{\mathbf{t}}+\mathbf{U}_{\mathbf{g}} \mathbf{h}_{\mathbf{t}-\mathbf{1}}+\mathbf{b}_{\mathbf{g}}\right) \\
& \mathbf{c}_{\mathbf{t}}=\mathbf{f}_{\mathbf{t}} \odot \mathbf{c}_{\mathbf{t}-\mathbf{1}}+\mathbf{i}_{\mathbf{t}} \odot \mathbf{g}_{\mathbf{t}} \\
& \mathbf{o}_{\mathbf{t}}=\sigma\left(\mathbf{W}_{\mathbf{o}} \mathbf{x}_{\mathbf{t}}+\mathbf{U}_{\mathbf{o}} \mathbf{h}_{\mathbf{t}-\mathbf{1}}+\mathbf{V}_{\mathbf{o}} \mathbf{c}_{\mathbf{t}}+\mathbf{b}_{\mathbf{o}}\right) \\
& \mathbf{h}_{\mathbf{t}}=\mathbf{o}_{\mathbf{t}} \odot \tanh \left(\mathbf{c}_{\mathbf{t}}\right)
\end{aligned}
$$

Here $\mathbf{x}_{\mathbf{t}}$ is the word embedding of word $w_{t}, \sigma$ denotes the sigmoid function, $\odot$ is element-wise multiplication. $\mathbf{W}_{\mathbf{i}}, \mathbf{U}_{\mathbf{i}}, \mathbf{V}_{\mathbf{i}}, \mathbf{b}_{\mathbf{i}}, \mathbf{W}_{\mathrm{g}}, \mathbf{U}_{\mathrm{g}}, \mathbf{b}_{\mathrm{g}}, \mathbf{W}_{\mathbf{o}}, \mathbf{U}_{\mathbf{o}}$, $\mathbf{V}_{\mathbf{o}}$ and $\mathbf{b}_{\mathbf{o}}$ are LSTM parameters.

\footnotetext{
${ }^{1}$ Most sentiment lexicons assign a negative score to the word "not".
} 
We apply a bidirectional extension of LSTM (BiLSTM) (Graves and Schmidhuber, 2005; Graves et al., 2013), shown in Figure 2, to encode the input sentence $s$ both left-to-right and right-to-left. The BiLSTM model maps each word $w_{t}$ to a pair of hidden vectors $\mathbf{h}_{\mathbf{t}}^{\mathbf{L}}$ and $\mathbf{h}_{\mathbf{t}}^{\mathbf{R}}$, which denote the hidden vector of the left-to-right LSTM and right-toleft LSTM, respectively. We use different parameters for the left-to-right LSTM and the right-to-left LSTM. These state vectors are used as features for calculating the sentiment weights $\gamma$.

In addition, we append a sentence end marker $w_{<e>}$ to the left-to-right LSTM and a sentence start marker $w_{<s>}$ to the right-to-left LSTM. The hidden state vector of $w_{<s>}$ and $w_{<e>}$ are denoted as $\mathbf{h}_{\mathbf{s}}^{\mathbf{R}}$ and $\mathbf{h}_{\mathbf{e}}^{\mathbf{L}}$, respectively.

\subsection{Output Layer}

The base score. Given a lexicon word $w_{j_{t}}$ in the sentence $s\left(w_{j_{t}} \in D\right)$, we use the hidden state vectors $\mathbf{h}_{\mathbf{j}_{\mathbf{t}}}^{\mathbf{L}}$ and $\mathbf{h}_{\mathbf{j}_{\mathbf{t}}}^{\mathbf{R}}$ in the feature layer to calculate a weight value $\tau_{j_{t}}$. As shown in Figure 3, a two-layer neural network is used to induce $\tau_{j_{t}}$. In particular, a hidden layer combines $\mathbf{h}_{\mathbf{t}}^{\mathbf{L}}$ and $\mathbf{h}_{\mathbf{t}}^{\mathbf{R}}$ using a nonlinear tanh activation

$$
\mathbf{p}_{\mathbf{j}_{\mathbf{t}}}^{\mathbf{s}}=\tanh \left(\mathbf{W}_{\mathbf{p s}}^{\mathbf{L}} \mathbf{h}_{\mathbf{j} \mathbf{t}}^{\mathbf{L}}+\mathbf{W}_{\mathbf{p s}}^{\mathbf{R}} \mathbf{h}_{\mathbf{j}_{\mathbf{t}}}^{\mathbf{R}}+\mathbf{b}_{\mathbf{p s}}\right)
$$

The resulting hidden vector $\mathbf{p}_{\mathbf{j} \mathbf{t}}^{\mathbf{s}}$ is then mapped into $\tau_{j_{t}}$ using another tanh layer.

$$
\tau_{j_{t}}^{s}=2 \tanh \left(\mathbf{W}_{\mathbf{p w}} \mathbf{p}_{\mathbf{j}_{\mathbf{t}}}^{\mathbf{s}}+\mathbf{b}_{\mathbf{p w}}\right)
$$

We choose the $2 \tanh$ function to make the learned weights conceptually useful. The factor 2 is introduced for modelling the effect of intensification. Since the range of tanh function is $[-1,1]$, the range of $2 \tanh$ is $[-2,2]$. Intuitively, a weight value of 1 maps the word sentiment directly to the sentence sentiment, such as the weight for "good" in "This is good". A weight value in $(1,2]$ represents intensification, such as the weight for "bad" in "very bad". Similarly, a weight value in $(0,1)$ represents weakening, and a weight in $(-2,0)$ represents various scales of negations.

Given all lexicon words $w_{j_{t}}^{D}$ in the sentence, we calculate a base score for the sentence

$$
S_{\text {base }}=\frac{\sum_{t=1}^{m} \tau_{j_{t}} \operatorname{score}\left(w_{j_{t}}^{D}\right)}{m}
$$

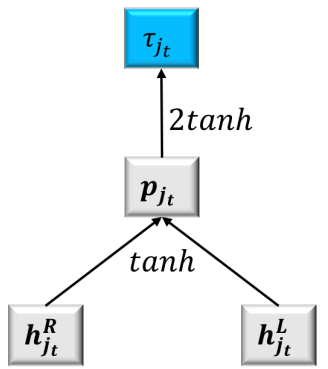

Figure 3: Weight score calculation.

By averaging the score of each word, the resulting $S_{\text {base }}$ is confined to $[-2 \alpha, 2 \alpha]$, where $\alpha$ is the maximum absolute value of word sentiment. In the above equations, $\mathbf{W}_{\mathbf{p s}}^{\mathbf{L}}, \mathbf{W}_{\mathbf{p s}}^{\mathbf{R}}, \mathbf{b}_{\mathbf{p s}}, \mathbf{W}_{\mathbf{p w}}$ and $\mathbf{b}_{\mathbf{p w}}$ are model parameters.

The bias score. We use the same neural network structure in Figure 3 to calculate the overall bias of the input sentence. The input to the neural network includes $\mathbf{h}_{\mathrm{s}}^{\mathbf{R}}$ and $\mathbf{h}_{\mathbf{e}}^{\mathbf{L}}$, and the output is a bias score $S_{\text {bias }}$. Intuitively, the calculation of $S_{\text {bias }}$ relies on information of the full sentence. $\mathbf{h}_{\mathrm{s}}^{\mathbf{R}}$ and $\mathbf{h}_{\mathrm{e}}^{\mathbf{L}}$ are chosen because they have commonly been used in the research literature to represent overall sentential information (Graves et al., 2013; Cho et al., 2014).

We use a dedicated set of parameters for calculating the bias, where

$$
\mathbf{p}^{\mathbf{B}}=\tanh \left(\mathbf{W}_{\mathbf{p b}}^{\mathbf{L}} \mathbf{h}_{\mathbf{e}}^{\mathbf{L}}+\mathbf{W}_{\mathbf{p b}}^{\mathbf{R}} \mathbf{h}_{\mathbf{s}}^{\mathbf{R}}+\mathbf{b}_{\mathbf{p b}}\right)
$$

and

$$
S_{\text {bias }}=2 \tanh \left(\mathbf{W}_{\mathbf{b}} \mathbf{p}^{\mathbf{B}}+\mathbf{b}_{\mathbf{p}}\right)
$$

$\mathbf{W}_{\mathbf{p b}}^{\mathbf{L}}, \mathbf{W}_{\mathbf{p b}}^{\mathbf{R}}, \mathbf{b}_{\mathbf{p b}}, \mathbf{W}_{\mathbf{b}}$ and $\mathbf{b}_{\mathbf{p}}^{\mathbf{L}}$ are parameters.

\subsection{Final Score Calculation}

The base $S_{b a s e}$ and bias $S_{\text {bias }}$ are linearly interpolated to derive the final sentiment value for the sentence $s$.

$$
\operatorname{Score}(s)=\lambda S_{\text {base }}+(1-\lambda) S_{\text {bias }}
$$

$\lambda \in[0,1]$ reflects the relative importance of the base score in the sentence. It offers a new degree of model flexibility, and should be calculated for each sentence specifically. We use the attention model (Bahdanau et al., 2014) to this end. In particular, the base score features $\mathbf{h}_{\mathbf{t}}^{\mathbf{L}} / \mathbf{h}_{\mathbf{t}}^{\mathbf{R}}$ and the bias score features $\mathbf{h}_{\mathbf{e}}^{\mathbf{L}} / \mathbf{h}_{\mathrm{s}}^{\mathbf{R}}$ are combined in the calculation

$$
\lambda=\sigma\left(\mathbf{W}_{\mathbf{s} \lambda} \mathbf{h}_{\text {base }}+\mathbf{W}_{\mathbf{b} \lambda} \mathbf{h}_{\text {bias }}+\mathbf{b}_{\lambda}\right)
$$


where

$$
\mathbf{h}_{\text {bias }}=\mathbf{h}_{\mathbf{e}}^{\mathbf{L}} \oplus \mathbf{h}_{\mathbf{s}}^{\mathbf{R}}
$$

and

$$
\mathbf{h}_{\text {base }}=\frac{\sum_{t=1}^{m} \mathbf{h}_{\mathbf{j} \mathbf{t}}^{\mathbf{L}} \oplus \mathbf{h}_{\mathbf{j}_{\mathbf{t}}}^{\mathbf{R}}}{m}
$$

Here $\sigma$ denotes the sigmoid activation function and $\oplus$ denotes vector concatenation. $\mathbf{W}_{\mathbf{s} \lambda}, \mathbf{W}_{\mathbf{b} \lambda}$ and $\mathbf{b}_{\lambda}$ are model parameters.

The final score of the sentence is

$$
\begin{aligned}
\operatorname{Score}(s) & =\lambda S_{\text {base }}+(1-\lambda) S_{\text {bias }} \\
& =\frac{\lambda}{m} \sum_{t=1}^{m} \tau_{j_{t}} \operatorname{score}\left(w_{j_{t}}^{D}\right)+(1-\lambda) S_{\text {bias }}
\end{aligned}
$$

This corresponds to the original Equation 1 by $\gamma_{j_{t}}=$ $\frac{\lambda}{m} \tau_{j_{t}}$ and $b=(1-\lambda) S_{\text {bias }}$.

\subsection{Training and Testing}

Our training data contains two different settings. The first is binary sentiment classification. In this task, every sentence $s_{i}$ is annotated with a sentiment label $l_{i}$, where $l_{i}=0$ and $l_{i}=1$ to indicate negative and positive sentiment, respectively. We apply logistic regression on the output layer. Denote the probability of a sentence $s_{i}$ being positive and negative as $p_{s_{i}}^{1}$ and $p_{s_{i}}^{0}$ respectively. $p_{s_{i}}^{0}$ and $p_{s_{i}}^{1}$ are estimated as

$$
\begin{aligned}
& p_{s_{i}}^{1}=\sigma\left(\operatorname{Score}\left(s_{i}\right)\right) \\
& p_{s_{i}}^{0}=1-p_{s_{i}}^{1}
\end{aligned}
$$

Suppose that there are $N$ training sentences, the loss function over the training set is defined as

$$
L(\boldsymbol{\Theta})=-\sum_{i=1}^{N} \log p_{s_{i}}^{l_{i}}+\frac{\lambda_{r}}{2}\|\boldsymbol{\Theta}\|^{2},
$$

where $\Theta$ is the set of model parameters. $\lambda_{r}$ is a parameter for $\mathrm{L} 2$ regularization.

The second setting is multi-class classification. In this task, every sentence $s_{i}$ is assigned a sentiment label $l_{i}$ from 0 to 4 , which represent very negative, negative, neutral, positive and very positive, respectively. We apply least square regression on the output layer. Since the output range of $2 \tanh$ is [-2, 2], the value of the base score and the bias score both belongs to $[-2,2]$. The final score is a weighted sum of the base score and the bias score, also belonging to $[-2,2]$. However, the gold sentiment label ranges

\begin{tabular}{l|c|c|c}
\hline & Positive & Negative & Total \\
\hline Train & 3,009 & 1,187 & 4,196 \\
Dev & 483 & 283 & 766 \\
Test & 1,313 & 490 & 1,803 \\
\hline
\end{tabular}

Table 1: Statistics of the Twitter dataset.

\begin{tabular}{c|c|c|c|c}
\hline Task & Label & $\begin{array}{c}\text { Training } \\
\text { Sentences }\end{array}$ & $\begin{array}{c}\text { Dev } \\
\text { Sentences }\end{array}$ & $\begin{array}{c}\text { Test } \\
\text { Sentences }\end{array}$ \\
\hline \multirow{5}{*}{ 5-class } & -2 & 1,092 & 139 & 279 \\
& -1 & 2,218 & 289 & 633 \\
& 0 & 1,624 & 229 & 389 \\
& 1 & 2,322 & 279 & 510 \\
& 2 & 1,288 & 165 & 399 \\
\hline \multirow{2}{*}{ 2-class } & 0 & 3,310 & 444 & 909 \\
& 1 & 3,610 & 428 & 912 \\
\hline
\end{tabular}

Table 2: Statistics of SST.

from 0 to 4 . We add an offset -2 to every gold sentiment label to both adapt our model to the training data and to increase the interpretability of the learned weights. The loss function for this problem is then defined as

$$
L(\boldsymbol{\Theta})=\sum_{i=1}^{N}\left(\operatorname{Score}\left(s_{i}\right)-l_{i}\right)^{2}+\frac{\lambda_{r}}{2}\|\boldsymbol{\Theta}\|^{2}
$$

During testing, we predict the sentiment label $l_{i}^{*}$ of a sentence $s_{i}$ by

$$
l_{i}^{*}=\left\{\begin{aligned}
-2 & \text { if } \operatorname{Score}\left(s_{i}\right) \leq-1.5 \\
-1 & \text { if }-1.5<\operatorname{Score}\left(s_{i}\right) \leq-0.5 \\
0 & \text { if }-0.5<\operatorname{Score}\left(s_{i}\right) \leq 0.5 \\
1 & \text { if } 0.5<\operatorname{Score}\left(s_{i}\right) \leq 1.5 \\
2 & \text { if } \operatorname{Score}\left(s_{i}\right)>1.5
\end{aligned}\right.
$$

\section{Experiments}

\subsection{Experimental Settings}

Data. We test our model on three datasets, including a dataset on Twitter sentiment classification, a dataset on movie review and a dataset with mixed domains. The Twitter dataset is taken from SemEval 2013 (Nakov et al., 2013). We downloaded the dataset according to the released ids. The statistics of the dataset are shown in Table 1.

The movie review dataset is Stanford Sentiment Treebank $^{2}$ (SST) (Socher et al., 2013). For each sentence in this treebank, a corresponding constituent

\footnotetext{
${ }^{2}$ http://nlp.stanford.edu/sentiment/index.html
} 


\begin{tabular}{l|c|c|c|c|c}
\hline Polarity & books & dvds & electronics & music & videogames \\
\hline Positive & 19 & 19 & 19 & 20 & 20 \\
Negative & 29 & 20 & 19 & 20 & 20 \\
\hline
\end{tabular}

Table 3: Document distribution of the mixed domain dataset.

tree is given. Each internal constituent node is annotated with a sentiment label ranging from 0 to 4 . We follow Socher et al. (2011) and Li et al. (2015) to perform five-class and binary classification, with the data statistics being shown in Table 2 .

In order to examine cross-domain robustness, we apply our model on a product review corpus (Täckström and McDonald, 2011), which contains 196 documents covering 5 domains: books, dvds, electronics, music and videogames. The document distribution is listed in Table 3.

Lexicons. We use four sentiment lexicons, namely TS-Lex, S140-Lex, SD-Lex and SWN-Lex. TS-Lex ${ }^{3}$ is a large-scale sentiment lexicon built from Twitter by Tang et al. (2014a) for learning sentiment-specific phrase embeddings. S140-Lex ${ }^{4}$ is the Sentiment140 lexicon, which is built from point-wise mutual information using distant supervision (Go et al., 2009; Mohammad et al., 2013).

SD-Lex is built from SST. We construct a sentiment lexicon from the training set by excluding all neutral words and adding the aforementioned offset -2 to each entry. SWN-Lex is a sentiment lexicon extracted from SentimentWordNet3.0 (Baccianella et al., 2010). For words with different partof-speech tags, we keep the minimum negative score or the maximum positive score. The original score in the SentimentWordNet3.0 is a probability value between 0 and 1 , and we scale it to $[-2,2]^{5}$.

When building these lexicons, we only use the sentiment scores for unigrams. Ambiguous words are discarded. Both TS-Lex and S140-Lex are Twitter-specific sentiment lexicons. They are used in the Twitter sentiment classification task. SD-Lex and SWN-Lex are exploited for the Stanford dataset. The statistics of lexicons are listed in Table 4.

\footnotetext{
${ }^{3}$ http://ir.hit.edu.cn/ dytang/paper/14coling/data.zip

${ }^{4} \mathrm{http}: / /$ saifmohammad.com/Lexicons/Sentiment140Lexicon-v0.1.zip

${ }^{5}$ Taboada et al. (2011) also mentioned two methods to derive sentiment score for a sentiment word from SentimentWordNet. We leave them for future work.
}

\begin{tabular}{l|c|c|c}
\hline Lexicon & Positive & Negative & Total \\
\hline SD-Lex & 2,547 & 2,448 & 4,995 \\
SWN-Lex & 15,568 & 17,412 & 32,980 \\
\hline TS-Lex & 33,997 & 32,026 & 66,023 \\
S140-Lex & 24,156 & 38,312 & 62,468 \\
\hline
\end{tabular}

Table 4: Statistics of sentiment lexicons.

\subsection{Implementation Details}

We implement our model based on the CNN toolkit. ${ }^{6}$ Parameters are optimized using stochastic gradient descent with momentum (Sutskever et al., 2013). The decay rate is 0.1 . For initial learning rate, L2 and other hyper-parameters, we adopt the default values provided by the $\mathrm{CNN}$ toolkits. We select the best model parameter according to the classification accuracy on the development set.

For the Twitter data, we use the glove.twitter. $27 B^{7}$ as pretrained word embeddings. For the Stanford dataset, following $\mathrm{Li}$ et al. (2015), we use glove. 840 B.300d $d^{8}$ as pretrained word embeddings. Words that do not exist in both the training set and the pretrained lookup table are treated as outof-vocabulary (OOV) words. Following Dyer et al. (2015), singletons in the training data are randomly mapped to UNK with a probability $p_{u n k}$ during training. We set $p_{u n k}=0.1$. All word embeddings are fine-tuned. We use dropout (Srivastava et al., 2014) in the input layer to prevent overfitting during training.

One-layered BiLSTM is used for all tasks. The dimension of the hidden vector in LSTM is 150 . The size of the second layer in Figure 3 is 64 .

\subsection{Development Results}

Table 5 shows results on the Twitter development set. Bi-LSTM is our model using the bias score $S_{\text {bias }}$ only, which is equivalent to bidirectional LSTM model of Li et al. (2015) and Tai et al. (2015), since they use same features and only differ in the output layer. Bi-LSTM+avg.lexicon is a baseline model integrating the average sentiment scores of lexicon words as a feature, and Bi-LSTM+flex.lexicon is our final model, which considers both the Bi-LSTM score $\left(S_{\text {bias }}\right)$ and the context-sensitive score $\left(S_{\text {base }}\right)$.

\footnotetext{
${ }^{6}$ https://github.com/clab/cnn

${ }^{7}$ http://nlp.stanford.edu/data/glove.twitter.27B.zip

${ }^{8} \mathrm{http}: / /$ nlp.stanford.edu/data/glove.840B.300d.zip
} 


\begin{tabular}{l|c|c}
\hline Method & Dict & Dev(\%) \\
\hline Bi-LSTM & None & 84.2 \\
Bi-LSTM+avg.lexicon & S140-Lex & 84.9 \\
Bi-LSTM+flex.lexicon & S140-Lex & $\mathbf{8 6 . 4}$ \\
\hline
\end{tabular}

Table 5: Results on the Twitter development set.

\begin{tabular}{l|c}
\hline Method & Test $(\%)$ \\
\hline SVM6 (Zhu et al., 2014) & 78.5 \\
Tang et al. (2014a) & 82.4 \\
\hline Bi-LSTM & 86.7 \\
Bi-LSTM + TS-Lex & 87.6 \\
Bi-LSTM + S140-Lex & $\mathbf{8 8 . 0}$
\end{tabular}

Table 6: Results on the Twitter test set.

Bi-LSTM+avg.lexicon improves the classification accuracy over Bi-LSTM by 0.7 point, which shows the usefulness of sentiment lexicons to recurrent neural models using a vanilla method. It is consistent with previous research on discrete models. By considering context-sensitive weighting for sentiment words Bi-LSTM+flex.lexicon further outperforms Bi-LSTM+avg.lexicon, improving the accuracy by 1.5 points $(84.9 \rightarrow 86.4)$, which demonstrates the strength of context-sensitive scoring. Base on the development results, we use BiLSTM+flex.lexicon for the remaining experiments.

\subsection{Main Results}

Twitter. Table 6 shows results on the Twitter test set. SVM6 is our implementation of Zhu et al. (2014), which extracts six types of manual features from TSLex for SVM classification. The features include: (1) the number of sentiment words in the sentence; (2) the total sentiment scores of the sentence; (3) the maximum sentiment score; (4) the total positive and negative sentiment scores; (5) the sentiment score of the last word in the sentence. The system of Tang et al. (2014a) is a state-of-the-art system that extracts various manually designed features from TSLex, such as bag-of-words, term frequency, parts-ofspeech, the sum of sentiment scores of all words in a tweet, etc, for SVM. The Bi-LSTM rows are our final models with different lexicons.

Both SVM6 and Tang et al. (2014a) exploit discrete features. Compared to them, Bi-LSTM gives better accuracies without using lexicons, which demonstrates the relative strength of deep neural network for sentiment analysis. Compared with Tang et al. (2014a), our Bi-LSTM+TS-Lex model improves

\begin{tabular}{lcc}
\hline Method & 5-class 2-class \\
\hline RAE (Socher et al., 2011) & 43.2 & 82.4 \\
MV-RNN (Socher et al., 2012) & 44.4 & 82.9 \\
RNTN (Socher et al., 2013) & 45.7 & 85.4 \\
DRNN (Irsoy and Cardie, 2014) & 49.8 & 88.6 \\
Dependency TreeLSTM (Tai et al., 2015) & 48.4 & 85.7 \\
Constituency TreeLSTM (Tai et al., 2015) 51.0 & 88.0 \\
Constituency TreeLSTM (Li et al., 2015) & 50.4 & 86.7 \\
S-LSTM (Zhu et al., 2015) & 50.1 & - \\
LSTM-RNN (Le and Zuidema, 2015) & 49.9 & 88.0 \\
CNN-non-static (Kim, 2014) & 48.0 & 87.2 \\
CNN-multichannel (Kim, 2014) & 47.4 & 88.1 \\
DCNN (Kalchbrenner et al., 2014) & 48.5 & 86.8 \\
Paragraph-Vec (Le and Mikolov, 2014) & 48.7 & 87.8 \\
NBoW (Kalchbrenner et al., 2014) & 42.4 & 80.5 \\
SVM (Socher et al., 2013) & 40.7 & 79.4 \\
\hline BiLSTM (Tai et al., 2015) & 49.1 & 87.5 \\
BiLSTM (Li et al., 2015) & 49.8 & 86.7 \\
Hier-Sequence (Li et al., 2015) & 50.7 & 86.9 \\
\hline Bi-LSTM+SD-Lex & 50.0 & 88.1 \\
Bi-LSTM+SWN-Lex & $\mathbf{5 1 . 1}$ & $\mathbf{8 9 . 2}$ \\
\hline
\end{tabular}

Table 7: Results on SST. 5-class shows fine-grained classification. The last block lists our results.

the sentiment classification accuracy from 82.4 to 87.6 , which again shows the strength of contextsensitive features. S140-Lex gives slight improvements over TS-Lex.

SST. Table 7 shows the results on SST. We include various results of recursive (the first block), convolutional (the second block), and sequential LSTM models (the fourth block). These neural models give the recent state-of-the-art on this dataset. Our method achieves highly competitive accuracies. In particular, compared to sequential LSTMs, our best model gives the top result both on the binary and fine-grained classification task. This shows the usefulness of lexicons to neural models. In addition, SWN-Lex gives better results compared with SDLex. This is intuitive because SD-Lex is a smaller lexicon compared to SWN-Lex (4,999 entries v.s. 32,980 entries). SD-Lex does not bring external knowledge to this dataset, while SWN-Lex does.

Cross-domain Results. Lexicon-based methods can be robust for cross-domain sentiment analysis (Taboada et al., 2011). We test the robustness of our model in the mixed domain dataset of product reviews (Täckström and McDonald, 2011). This dataset contains document level sentiments. We take the majority voting strategy to transform sentiment 


\begin{tabular}{c|c|c|c|c|c|c|c|c}
\hline Model & Train & Test & Books & Dvds & Electronics & Music & Videogames & Average \\
\hline Bi-LSTM & None & None & 71.79 & 89.74 & 65.79 & 95 & $\mathbf{8 5}$ & 81.63 \\
Bi-LSTM+flex.lexicon & SD-Lex & SD-Lex & 76.92 & 84.62 & $\mathbf{7 8 . 9 5}$ & 92.5 & 80 & 82.65 \\
Bi-LSTM+flex.lexicon & SD-Lex & SWN-Lex & 82.05 & $\mathbf{9 2 . 3 1}$ & 73.68 & 92.5 & 80 & 84.18 \\
Bi-LSTM+flex.lexicon & SWN-Lex & SWN-Lex & $\mathbf{8 4 . 6 2}$ & $\mathbf{9 2 . 3 1}$ & 68.42 & $\mathbf{1 0 0}$ & $\mathbf{8 5}$ & $\mathbf{8 6 . 2 2}$ \\
\hline
\end{tabular}

Table 8: Cross-domain sentiment analysis. Training domain is movie review.

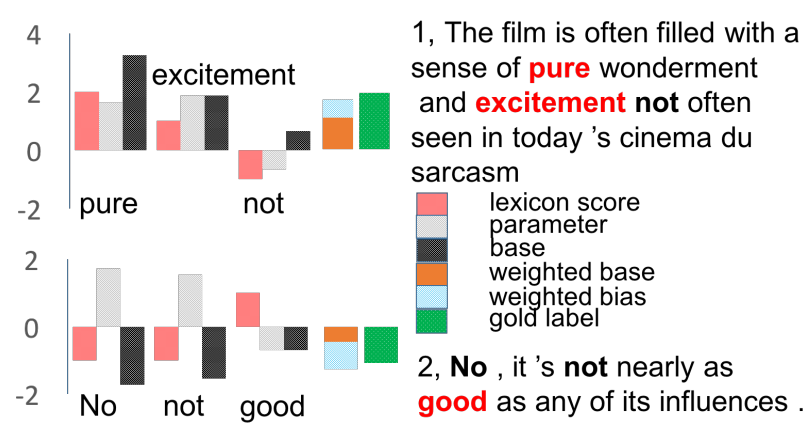

Figure 4: Sentiment composition examples.

of sentences to the document level. We compare the effects of different lexicons over a baseline $\mathrm{Bi}$ LSTM trained on SST (movie domain).

Table 8 shows the results. Introducing the sentiment lexicons SD-Lex and SWN-Lex consistently improves the classification accuracy across five domains compared with the baseline Bi-LSTM model. When trained and tested using the same lexicon, SWN-Lex gives better performances on three out of five domains. SD-Lex gives better results only on Electronics. This shows that the results are sensitive to the domain of the sentiment lexicon, which is intuitive.

We also investigate a model trained using SDLex but tested by replacing SD-Lex with SWN-Lex. This is to examine the generalizability of a sourcedomain model on different target domains by plugging in relevant domain-specific lexicons, without being retrained. Results show that the mode still outperforms the SD-Lex lexicon on two out of five domains, but is less accurate than full retraining using SWN-Lex.

\subsection{Discussion}

Figure 4 shows the details of sentiment composition for two sentences in the SST, learned automatically by our model. For the first sentence, the three subjective words in the lexicon "pure", "excitement"

\begin{tabular}{c|l|c|c}
\hline ID & Sentence & Bi-LSTM & SWN-Lex \\
\hline 1 & $\begin{array}{l}\text { The issue of faith is not } \\
\text { explored very deeply }\end{array}$ & 0 & -1 \\
\hline 2 & $\begin{array}{l}\text { Steers turns in a snappy } \\
\text { screenplay that curls at } \\
\text { the edges; it 's so clever } \\
\text { you want to hate it. }\end{array}$ & 2 & 1 \\
\hline 3 & $\begin{array}{l}\text { A film so tedious that it is } \\
\text { impossible to care whether } \\
\text { that boast is true or not. }\end{array}$ & -2 & -1 \\
\hline
\end{tabular}

Table 9: Example predictions made by the Bi-LSTM model and our Bi-LSTM+SWN-Lex model for fine-grained classification task. Red words and blue words are positive and negative entries in the SentimentWordNet3.0 lexicon, respectively.

and "not" receives weights of 1.6, 1.9 and -0.6 , respectively, and the overall bias of the sentence is positive. A $\lambda$ value (0.58) that slightly biases towards the base score leads to a final sentiment score is 1.8 , which is close to the gold label 2 .

In the second example, both negation words received positive weight values, and the bias over the sentence is negative. A $\lambda(0.3)$ value that biases towards the bias score results in a final score of -1.2 , which is close to the gold label -1 . These results demonstrate the capacity of the model to decide how word-level sentiments composite according to sentence-level context.

Table 9 shows three sentences in the Stanford test set which are incorrectly classified by $\mathrm{Bi}$ LSTM model, but correctly labeled by our $\mathrm{Bi}$ LSTM+SWN-Lex model. These examples show that our model is more sensitive to contextdependent sentiment changes, thanks to the use of lexicons as a basis.

\section{Conclusion}

We proposed a conceptually-simple, yet empirically effective method of introducing sentiment lexicon features to state-of-the-art LSTM models for sentiment analysis. Compared to the simple averag- 
ing method in traditional bag-of-word models, our system leverages the strength of semantic feature learning by LSTM models to calculate a contextdependent weight for each word given an input sentence. The method gives competitive results on various sentiment analysis benchmarks. In addition, thanks to the use of lexicons, our model can improve the cross-domain robustness of recurrent neural models for sentiment analysis.

\section{Acknowledgments}

Yue Zhang is the corresponding author. Thanks to anonymous reviewers for their helpful comments and suggestions. Yue Zhang is supported by NSFC61572245 and T2MOE201301 from Singapore Ministry of Education.

\section{References}

Apoorv Agarwal, Boyi Xie, Ilia Vovsha, Owen Rambow, and Rebecca Passonneau. 2011. Sentiment analysis of twitter data. In Proceedings of the Workshop on Languages in Social Media, pages 30-38.

Stefano Baccianella, Andrea Esuli, and Fabrizio Sebastiani. 2010. Sentiwordnet 3.0: An enhanced lexical resource for sentiment analysis and opinion mining. In Proceedings of LREC, volume 10, pages 2200-2204.

Dzmitry Bahdanau, Kyunghyun Cho, and Yoshua Bengio. 2014. Neural machine translation by jointly learning to align and translate. CoRR, abs/1409.0473.

Erik Cambria. 2016. Affective computing and sentiment analysis. IEEE Intelligent Systems, 31(2):102-107.

Kyunghyun Cho, Bart van Merrienboer, Caglar Gulcehre, Dzmitry Bahdanau, Fethi Bougares, Holger Schwenk, and Yoshua Bengio. 2014. Learning phrase representations using rnn encoder-decoder for statistical machine translation. In Proceedings of EMNLP, pages 1724-1734.

Yejin Choi and Claire Cardie. 2008. Learning with compositional semantics as structural inference for subsentential sentiment analysis. In Proceedings of EMNLP, pages 793-801.

Kerstin Denecke. 2009. Are sentiwordnet scores suited for multi-domain sentiment classification? In ICDIM, pages 1-6. IEEE.

Ann Devitt and Khurshid Ahmad. 2007. Sentiment polarity identification in financial news: A cohesionbased approach.

Xiaowen Ding, Bing Liu, and Philip S Yu. 2008. A holistic lexicon-based approach to opinion mining. In Pro- ceedings of the 2008 International Conference on Web Search and Data Mining, pages 231-240.

Li Dong, Furu Wei, Chuanqi Tan, Duyu Tang, Ming Zhou, and $\mathrm{Ke} \mathrm{Xu}$. 2014. Adaptive recursive neural network for target-dependent twitter sentiment classification. In Proceedings of ACL, pages 49-54.

Chris Dyer, Miguel Ballesteros, Wang Ling, Austin Matthews, and Noah A. Smith. 2015. Transitionbased dependency parsing with stack long short-term memory. In $A C L$.

Andrea Esuli and Fabrizio Sebastiani. 2006. Sentiwordnet: A publicly available lexical resource for opinion mining. In Proceedings of LREC, volume 6, pages 417-422.

Alec Go, Richa Bhayani, and Lei Huang. 2009. Twitter sentiment classification using distant supervision. CS224N Project Report, Stanford, 1:12.

Alex Graves and Jürgen Schmidhuber. 2005. Framewise phoneme classification with bidirectional lstm and other neural network architectures. Neural Networks, 18(5):602-610.

A. Graves, A. Mohamed, and G. Hinton. 2013. Speech recognition with deep recurrent neural networks.

Marco Guerini, Lorenzo Gatti, and Marco Turchi. 2013. Sentiment analysis: How to derive prior polarities from SentiWordNet. In Proceedings of EMNLP, pages 1259-1269.

Sepp Hochreiter and Jürgen Schmidhuber. 1997. Long short-term memory. Neural computation, 9(8):17351780 .

Minqing $\mathrm{Hu}$ and Bing Liu. 2004. Mining and summarizing customer reviews. In Proceedings of $K D D, \mathrm{KDD}$ '04, pages 168-177.

Ozan Irsoy and Claire Cardie. 2014. Deep recursive neural networks for compositionality in language. In Advances in Neural Information Processing Systems, pages 2096-2104.

Nal Kalchbrenner, Edward Grefenstette, and Phil Blunsom. 2014. A convolutional neural network for modelling sentences. In Proceedings of ACL, pages 655665.

Colm Kearney and Sha Liu. 2014. Textual sentiment in finance: A survey of methods and models. International Review of Financial Analysis, 33:171-185.

Soo-Min Kim and Eduard Hovy. 2004. Determining the sentiment of opinions. In Proceedings of the 20th international conference on Computational Linguistics, page 1367.

Yoon Kim. 2014. Convolutional neural networks for sentence classification. In Proceedings of EMNLP, pages 1746-1751.

Svetlana Kiritchenko, Xiaodan Zhu, and Saif M. Mohammad. 2014. Sentiment analysis of short informal texts. J. Artif. Intell. Res. (JAIR), 50:723-762. 
Quoc V. Le and Tomas Mikolov. 2014. Distributed representations of sentences and documents. CoRR, abs/1405.4053.

Phong Le and Willem Zuidema. 2015. Compositional distributional semantics with long short term memory. In Proceedings of the Fourth Joint Conference on Lexical and Computational Semantics, pages 10-19.

Jiwei Li, Minh-Thang Luong, Dan Jurafsky, and Eudard Hovy. 2015. When are tree structures necessary for deep learning of representations?

Pengfei Liu, Xipeng Qiu, Xinchi Chen, Shiyu Wu, and Xuanjing Huang. 2015. Multi-timescale long shortterm memory neural network for modelling sentences and documents. In Proceedings of EMNLP, pages 2326-2335.

Saif M. Mohammad, Svetlana Kiritchenko, and Xiaodan Zhu. 2013. Nrc-canada: Building the state-of-theart in sentiment analysis of tweets. In Proceedings of SemEval-2013, June.

Karo Moilanen and Stephen Pulman. 2007. Sentiment composition.

Richard Montague. 1974. Formal Philosophy: Selected Papers of Richard Montague. Ed. and with an Introd. by Richmond H. Thomason. Yale University Press.

Preslav Nakov, Sara Rosenthal, Zornitsa Kozareva, Veselin Stoyanov, Alan Ritter, and Theresa Wilson. 2013. Semeval-2013 task 2: Sentiment analysis in twitter. In Second Joint Conference on Lexical and Computational Semantics (*SEM), Volume 2: Proceedings of SemEval-2013, pages 312-320.

Thien Hai Nguyen and Kiyoaki Shirai. 2015. Phrasernn: Phrase recursive neural network for aspect-based sentiment analysis. In Proceedings of EMNLP.

Livia Polanyi and Annie Zaenen. 2006. Contextual valence shifters. In Computing attitude and affect in text: Theory and applications, pages 1-10.

Yafeng Ren, Yue Zhang, Meishan Zhang, and Donghong Ji. 2016. Context-sensitive twitter sentiment classification using neural network. In Proceedings of AAAI.

Richard Socher, Jeffrey Pennington, Eric H. Huang, Andrew Y. Ng, and Christopher D. Manning. 2011. Semi-Supervised Recursive Autoencoders for Predicting Sentiment Distributions. In Proceedings of EMNLP.

Richard Socher, Brody Huval, Christopher D. Manning, and Andrew Y. Ng. 2012. Semantic compositionality through recursive matrix-vector spaces. In Proceedings of EMNLP, pages 1201-1211.

Richard Socher, Alex Perelygin, Jean Wu, Jason Chuang, D. Christopher Manning, Andrew Ng, and Christopher Potts. 2013. Recursive deep models for semantic compositionality over a sentiment treebank. In Proceedings of EMNLP, pages 1631-1642.
Nitish Srivastava, Geoffrey Hinton, Alex Krizhevsky, Ilya Sutskever, and Ruslan Salakhutdinov. 2014. Dropout: A simple way to prevent neural networks from overfitting. The Journal of Machine Learning Research, 15(1):1929-1958.

Ilya Sutskever, James Martens, George Dahl, and Geoffrey Hinton. 2013. On the importance of initialization and momentum in deep learning. In Proceedings of the 30th international conference on machine learning (ICML-13), pages 1139-1147.

Maite Taboada, Julian Brooke, Milan Tofiloski, Kimberly Voll, and Manfred Stede. 2011. Lexicon-based methods for sentiment analysis. Computational linguistics, 37(2):267-307.

Oscar Täckström and Ryan McDonald. 2011. Discovering fine-grained sentiment with latent variable structured prediction models. In Advances in Information Retrieval, pages 368-374.

Kai Sheng Tai, Richard Socher, and Christopher D. Manning. 2015. Improved semantic representations from tree-structured long short-term memory networks. In Proceedings of ACL, pages 1556-1566.

Duyu Tang, Furu Wei, Bing Qin, Ming Zhou, and Ting Liu. 2014a. Building large-scale twitter-specific sentiment lexicon : A representation learning approach. In Proceedings of COLING, pages 172-182, August.

Duyu Tang, Furu Wei, Nan Yang, Ming Zhou, Ting Liu, and Bing Qin. 2014b. Learning sentiment-specific word embedding for twitter sentiment classification. In Proceedings of ACL, pages 1555-1565, June.

Peter Turney. 2002. Thumbs up or thumbs down? semantic orientation applied to unsupervised classification of reviews. In Proceedings of ACL.

Duy-Tin Vo and Yue Zhang. 2015. Target-dependent twitter sentiment classification with rich automatic features. In Proceedings of IJCAI, pages 1347-1353, July.

Xin Wang, Yuanchao Liu, Chengjie SUN, Baoxun Wang, and Xiaolong Wang. 2015. Predicting polarities of tweets by composing word embeddings with long short-term memory. In Proceedings of $A C L$, pages 1343-1353.

Theresa Wilson, Janyce Wiebe, and Paul Hoffmann. 2005. Recognizing contextual polarity in phrase-level sentiment analysis. In Proceedings of HLT-EMNLP.

Meishan Zhang, Yue Zhang, and Duy-Tin Vo. 2016. Gated neural networks for targeted sentiment analysis.

Xiaodan Zhu, Svetlana Kiritchenko, and Saif M Mohammad. 2014. Nrc-canada-2014: Recent improvements in the sentiment analysis of tweets.

Xiaodan Zhu, Parinaz Sobhani, and Hongyu Guo. 2015. Long short-term memory over tree structures. CoRR, abs/1503.04881. 